\section{Cylene takes pol position in cancer}

\section{By Tim Fulmer, Senior Writer}

Australian and U.S. researchers have shown that a small molecule inhibitor of RNA polymerase I blocked tumor growth in mouse models of lymphoma. ${ }^{1}$ Cylene Pharmaceuticals Inc. and the Peter MacCallum Cancer Centre plan to start a Phase I trial of the inhibitor in Australia this year.

During tumorigenesis, cancer cells produce abnormally high levels of rRNA to create the ribosomes necessary for rapid protein synthesis and cellular proliferation. ${ }^{2,3}$ Because the enzyme RNA polymerase I ( $\mathrm{Pol}$ I) drives the synthesis of rRNA, it has been hypothesized that inhibiting Pol I could be a way to indirectly block protein synthesis and prevent cancer cell proliferation.

The challenge has been figuring out how to target Pol I in cancer cells without impairing the function of the enzyme in healthy cells, in which it plays an essential role in normal protein synthesis.

In prior work, Cylene researchers used a cell-based screening assay to identify a small molecule that blocked rRNA synthesis by inhibiting Pol I. ${ }^{4}$ In the new work, they tested in vivo whether the same Pol I inhibitor, CX-5461, could selectively kill hematological cancer cells, which often show dysregulation of rRNA synthesis and ribosome biogenesis.

In cultured lymphoma cells isolated from tumor-bearing mice, CX-5461 induced apoptosis and increased cell death compared with vehicle. Moreover, the killing effect was more pronounced in cells expressing wild-type p53 than in cells expressing mutant p53 or no p53 at all, suggesting CX-5461 required the downstream activity of wild-type p53.

Additional cell culture studies revealed a mechanism whereby the inhibition of Pol I by CX-5461 led to the buildup of ribosomal proteins and the activation of $\mathrm{p} 53$, which then upregulated apoptotic pathways to kill cancer cells (see Figure 1, "Pol I inhibition in cancer").

Importantly, CX-5461 did not activate p53 and induce apoptosis in normal immune cells, confirming that the inhibitor's killing effects were selective for malignant cells.

In mice with established lymphoma expressing wild-type p53, a single dose of CX-5461 significantly decreased tumor burden in the lymph nodes compared with vehicle $(p<0.01)$. In a separate group of the same lymphoma mice, three doses of CX-5461 significantly prolonged survival and restored white blood cell counts.

To translate the findings to human disease, the researchers next evaluated CX-5461 in a panel of cell lines derived from human hematological cancers. Consistent with the mouse data, the inhibitor induced apoptosis in cell lines expressing wild-type p53 much more potently than in cells expressing mutant $\mathrm{p} 53$.

Finally, in mice transplanted with one of the human hematological cancer cell lines, once weekly CX-5461 decreased tumor volume compared with vehicle.

The team was led by Ross Hannan, associate professor of biochemistry and molecular biology at The University of Melbourne and head of the Oncogenic Signaling and Growth Control Program at Peter MacCallum, and Grant McArthur, cohead of the Cancer Therapeutics Program and director of the Melanoma \& Skin Cancer Service at Peter MacCallum. Denis Drygin, Cylene VP of biology, and William Rice, Cylene president and CEO, were co-principal investigators on the study.

The findings were published in Cancer Cell.

"The field has known for a while that cancer cells become addicted to ribosomal biogenesis and protein synthesis for their survival and proliferation," Davide Ruggero told SciBX. “This paper shows for the first time it is possible to directly target one of those processes without causing serious toxicity to healthy cells."

In 2011, Ruggero, associate professor of urology at the University of

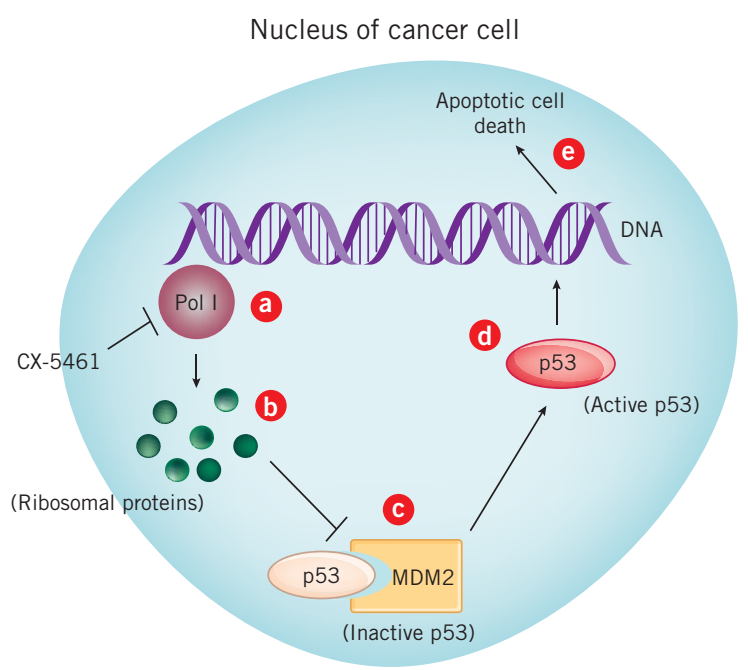

Figure 1. Pol I inhibition in cancer. Researchers from the Peter MacCallum Research Centre and Cylene Pharmaceuticals Inc. have published data in Cancer Cell showing that a small molecule inhibitor of RNA polymerase I (Pol I) reduced growth of hematological cancers in mice.

In lymphoma cells, the Pol I inhibitor CX-5461 blocked Pol I with low nanomolar potency [a], triggering a stress response involving the release of ribosomal proteins from the nucleolus [b], a subcompartment of the nucleus. The ribosomal proteins then blocked interactions between p53 and the p53 inhibitory protein mdm2 p53 binding protein homolog (MDM2; HDM2) [c], which in turn activated p53 [d] and allowed it to upregulate expression of proapoptotic genes, killing the cancer cells [e].

Cylene and Peter MacCallum plan to start a Phase I trial of CX-5461 to treat hematological malignancies this year. 
California, San Francisco, published in Cancer Cell that the protein synthesis regulator eukaryotic translation initiation factor $4 \mathrm{E}$ binding protein 1 (eIF4EBP1) is a key mediator of cancer cell growth and progression. ${ }^{5}$

\section{Activating p53}

The key advantage of CX-5461 is that it activates p53 through a downstream ribosomal stress pathway, which “doesn't cause global DNA damage, and thus the side effects of the drug are significantly reduced compared to standard cytotoxic therapies and radiation," said Hannan. In fact, CX-5461 is 300 -fold more potent than nutlins at killing lymphoma cell lines, he added.

Nutlins, small molecule antagonists of mdm2 p53 binding protein homolog (MDM2; HDM2) that result in activation of $\mathrm{p} 53$, were developed by Roche and are in Phase I testing to treat various cancers. In preclinical studies, nutlins induced apoptosis in blood cancers including acute myelogenous leukemia (AML) and cutaneous T cell lymphoma (CTCL) ${ }^{6,7}$ Roche declined to comment.

According to Klas Wiman, professor of oncology at the Karolinska Institute and cofounder of Aprea AB, the fact that CX-5461 works well in tumors that carry wild-type p53 but not so well in tumors that lack p53 or express mutant p53 is a potential limitation of the compound. "Targeting mutant p53-carrying tumors is a greater challenge, and here there is an urgent medical need, because mutant p53-carrying tumors are more resistant to conventional chemotherapy and have a worse prognosis," said Wiman.

Aprea's lead is PRIMA-1MET (APR-246), a small molecule that promotes the folding of mutant p53 into an active form. The compound is in Phase II testing to treat AML and chronic lymphocytic leukemia (CLL). In cultured cancer cells, PRIMA-1MET reactivated mutant p53 and triggered apoptotic cell death. ${ }^{8}$

CX-5461 "should be tested in a wider range of tumor types," said Wiman. He added that "it should be tested in ex vivo primary patient tumor cells to study its differential effects on tumors carrying wild-type versus mutant $\mathrm{p} 53$."

Cylene is continuing to look for genetic markers of sensitivity to CX-5461 in a wide variety of blood and solid cancers, Rice told SciBX.

Indeed, there is evidence that some solid tumors are sensitive to CX-5461, according to Hannan. "For example, preliminary studies have shown that a panel of ovarian cell lines exhibits a 1,000-fold range in sensitivity to CX-5461."

Meanwhile, Cylene will focus mainly on hematological cancers, which have a relatively low frequency of p53 mutations compared with many solid cancers ( $13 \%$ vs. $>40 \%) .{ }^{9,10}$

In the second half of this year, Cylene and Peter MacCallum researchers will start a Phase I trial of CX-5461 in Australia to treat hematological malignancies including advanced leukemia, lymphoma and multiple myeloma (MM).

The initial dose-escalation phase of the trial will include patients with any relapsed or refractory hematological malignancy, whereas the second phase will enrich for patients whose disease is wild type for p53, such as mixed-lineage leukemia, said Hannan.

Cylene has patents covering CX-5461 and the Cancer Cell findings that are available for licensing. Hannan did not disclose if Peter MacCallum has IP associated with any of the findings.

Fulmer, T. SciBX 5(30); doi:10.1038/scibx.2012.774

Published online Aug. 2, 2012

\section{REFERENCES}

1. Bywater, M.J. et al. Cancer Cell; published online July 10, 2012; doi:10.1016/j.ccr.2012.05.019

Contact: Ross D. Hannan, Peter MacCallum Cancer Centre, East Melbourne, Victoria, Australia e-mail: ross.hannan@petermac.org

2. Drygin, D. et al. Annu. Rev. Pharmacol. Toxicol. 50, 131-156 (2010)

3. White, R.J. Nat. Rev. Mol. Cell Biol. 6, 69-78 (2005)

4. Drygin, D. et al. Cancer Res. 71, 1418-1430 (2011)

5. Hsieh, A.C. et al. Cancer Cell 17, 249-261 (2010)

6. Kojima, K. et al. Blood 106, 3150-3159 (2005)

7. Manfé, V. et al. J. Invest. Dermatol. 132, 1487-1496 (2012)

8. Lambert, J.M.R. et al. Cancer Cell 15, 376-388 (2009)

9. Peller, S. \& Rotter, V. Hum. Mutat. 21, 277-284 (2003)

10. Olivier, M. et al. Cold Spring Harb. Perspect. Biol. 2, a001008; published online Nov. 11, 2009; doi:10.1101/cshperspect.a001008

COMPANIES AND INSTITUTIONS MENTIONED

Aprea AB, Stockholm, Sweden

Cylene Pharmaceuticals Inc., San Diego, Calif.

Karolinska Institute, Stockholm, Sweden

Peter MacCallum Cancer Centre, East Melbourne, Victoria,

Australia

Roche (SIX:ROG; OTCQX:RHHBY), Basel, Switzerland

University of California, San Francisco, Calif.

The University of Melbourne, Parkville, Victoria, Australia 